\title{
A Hybrid Approach for Fault Diagnosis of Railway Rolling Bearings Using STWD-EMD-GA-LSSVM
}

\author{
Dechen Yao, ${ }^{1,2}$ Jianwei Yang, ${ }^{1,2} \mathrm{Xi} \mathrm{Li}^{3}$ and Chunqing Zhao ${ }^{1,2}$ \\ ${ }^{1}$ School of Machine-Electricity and Automobile Engineering, Beijing University of Civil Engineering Architecture, Beijing 100044, China \\ ${ }^{2}$ Beijing Key Laboratory of Performance Guarantee on Urban Rail Transit Vehicles, Beijing University of Civil Engineering Architecture, \\ Beijing 100044, China \\ ${ }^{3}$ Subway Operation Technology Centre, Mass Transit Railway Operation Corporation Ltd., Beijing 102208, China
}

Correspondence should be addressed to Dechen Yao; yaodechen@bucea.edu.cn

Received 16 September 2015; Accepted 29 February 2016

Academic Editor: Yongjun Shen

Copyright (C) 2016 Dechen Yao et al. This is an open access article distributed under the Creative Commons Attribution License, which permits unrestricted use, distribution, and reproduction in any medium, provided the original work is properly cited.

Vibration signals resulting from railway rolling bearings are nonstationary by nature; this paper proposes a hybrid approach for the fault diagnosis of railway rolling bearings using segment threshold wavelet denoising (STWD), empirical mode decomposition (EMD), genetic algorithm (GA), and least squares support vector machine (LSSVM). The original signal is first denoised using STWD as a prefilter, which improves the subsequent decomposition into a number of intrinsic mode functions (IMFs) using EMD. Secondly, the IMF energy-torques are extracted as feature parameters. Concurrently, a GA is employed to optimize the LSSVM to improve the classification accuracy. Finally, the extracted features are used as inputs for classification by the GA-LSSVM. Actual railway rolling bearing vibration signals are used to experimentally verify the effectiveness of the proposed method. The results show that the novel method is effective and accurate for fault diagnosis of railway rolling bearings.

\section{Introduction}

Rolling bearings are one of the crucial components used in the railway sector, and bearing failure generally leads to serious damage for the railway. Hence, the fault diagnosis of railway rolling bearings is of great significance [1]. In fault diagnosis, the most important aspect is the feature extraction, which is employed to characterize the operating status of railway rolling bearings. Accurate and effective features can be easily used for automatic fault diagnosis in tandem with a neural network [2] or a relevance vector machine [3]. However, the nonlinear and nonstationary nature of acquired railway rolling bearing vibration signals and the existence of interferences caused by external factors both increase the difficulty of extracting features from the complex vibration signal. Over the last two decades, numerous fault diagnosis methods have been developed such as envelope analysis, short-time Fourier transform (STFT) [4], principal component analysis (PCA) [5], artificial neural network (ANN) [6], and genetic algorithm (GA) [7]. In this paper, a hybrid method for the fault diagnosis of railway rolling bearings is presented. The vibration signal from a bearing at an early stage of defect development is often masked by machine noise, making it difficult to detect the fault by vibration analysis techniques [8]; therefore, segment threshold wavelet denoising (STWD) is used as a prefilter for denoising. The vibration signal is then decomposed via empirical mode decomposition (EMD), which is a very reasonable approach for nonstationary signal analysis. EMD is used to extract the energy-torques of the intrinsic mode functions (IMFs) as feature parameters to be input into a least squares support vector machine (LSSVM) for classification. A GA is employed to search for optimal LSSVM parameters to ensure optimal adaptation in its global scope. Actual railway rolling bearing vibration signals are used to experimentally verify the effectiveness of the proposed method. The results show that the proposed method is effective and achieves a high recognition rate for fault diagnosis of railway rolling bearings.

The remainder of this paper is organized as follows. EMD and energy-torque feature extraction are discussed in Section 2. GA-LSSVM is described in Section 3. In Section 4, 
the method is validated experimentally. Finally, conclusions are drawn in Section 5.

\section{EMD and Energy-Torque Feature Extraction}

2.1. EMD. The EMD method proposed by Huang et al. [9] decomposes a signal into a number of IMFs and a single residue. Each IMF must satisfy the following conditions:

(1) Over the entire dataset, the number of extrema and the number of zero-crossings must either be equal or differ at most by one.

(2) At any point, the mean values of the envelopes defined by local maxima and by local minima are zero.

In accordance with this definition, any signal $x(t)$ can be decomposed as follows [10].

Step 1. Define $x(t)=x_{0}(t)$ and $r_{0}(t)=x_{0}(t)$.

Step 2. Define the maximum number of extracted IMFs.

Step 3. Identify all the local extrema of $x(t)$.

Step 4. Connect all local maxima and minima by a cubic spine as the upper envelope $E_{\max }(t)$ and the lower envelope $E_{\min }(t)$, respectively.

Step 5. Construct the mean of the upper and lower envelopes $m(t)=0.5\left(E_{\max }(t)+E_{\min }(t)\right)$.

Step 6. Define the detail (proto-IMF) as $d(t)=x(t)-m(t)$, and replace $x(t)$ by $d(t)$.

Step 7. Repeat Steps 3-6 until $d(t)$ meets IMF conditions (1) and (2) and the stoppage criterion of the sifting process is fulfilled; then derive the $i$ th $\operatorname{IMF}\left(c_{i}(t)\right)$ from $d(t)$ and replace $x(t)$ by $r_{i}(t)=r_{i-1}(t)-d(t)$.

Step 8. If the stoppage criterion of the signal's decomposition is fulfilled, then finish the decomposition process; otherwise, go to Step 3.

2.2. Energy-Torque Feature Extraction. The steps for energytorque feature extraction are as follows.

Step 1. STWD is used to filter the railway rolling bearing signals.

Step 2. The denoised vibration signals are decomposed into some number of IMFs via EMD, and the first $n$ IMFs, that is, $c_{i}(t), i=1,2,3, \ldots, n$, which include the most dominant fault energy, are chosen to extract the features.

Step 3. Calculate the energy-torque of every small time block, which, for a discrete signal, is given as

$$
E_{i}=\sum_{k=1}^{m}(k \cdot \Delta t)\left|c_{i}(k \cdot \Delta t)\right|^{2},
$$

where $m$ is the total number of sampling points $k$ and $\Delta t$ is the sampling period. Calculate the energy-torques $E_{1}, E_{2}, \ldots, E_{n}$ for all respective $c_{i}(t), i=1,2,3, \ldots, n$, based on (1).

Step 4. Construct the feature vector $\mathbf{T}$ from $E_{1}, E_{2}, \ldots, E_{n}$ :

$$
\mathbf{T}=\left[\begin{array}{llll}
E_{1} & E_{2} & \cdots & E_{n}
\end{array}\right] .
$$

When $E_{i}$ become large, normalize $\mathbf{T}$ as follows:

$$
\mathbf{T}^{\prime}=\left[\frac{E_{1}}{E}, \frac{E_{2}}{E}, \ldots, \frac{E_{n}}{E}\right],
$$

where

$$
E=\left(\sum_{i=1}^{n}\left|E_{i}\right|^{2}\right)^{1 / 2}
$$

The $i$ th IMF energy-torque is then calculated as follows [11]:

$$
E_{i}=\int_{-\infty}^{+\infty}\left|c_{i}(t)\right|^{2} d t
$$

\section{GA-LSSVM Algorithm}

3.1. GA. GA is a method proposed by Holland [12] for providing solutions to optimization and learning problems and is based freely on several features of biological evolution [13]. The algorithm begins with the initialization of a population of candidate solutions of which each is comprised of alterable properties denoted as chromosomes or a genotype. The initialized population is then evolved using genetic operators, giving, as in nature, more reproductive opportunities to the most highly fit chromosomes (i.e., those providing the best solution to the problem considered based on a fitness function) [14]. The GA applies selection, crossover, and mutation operators to construct fitter solutions and further processes the population by replacing unsuitable candidates according to the fitness function.

(1) Initialization of Population. Set the population scale and generate initial population including individuals with the number $N$. Set the range of data and select linear interpolation function [15] to generate real vectors as the individuals of GA.

(2) Determination of Fitness Function. Fitness function is a good standard which will effectively evaluate the adaptability to environment of individuals in population.

(3) Selection. The paper uses roulette wheel selection [16] to determine the probability by which the individual will be selected. The roulette wheel selection is a kind of selecting strategy for individual based on the fitness proportion. The formula of selection probability is shown as follows:

$$
p_{i}=\frac{f_{i}}{\sum_{i=1}^{N} f_{i}}, \quad i=1,2, \ldots, N,
$$

where $N$ is the population scale and $f_{i}$ is the reciprocal of individual fitness.

(4) Crossover and Mutation. To generate new population, GA takes the operations of crossover and mutation to deal 
with current population. As a consequence, probabilities of crossover and mutation are two important parameters which will have a great effect on the performance and property of convergence of GA. Different from traditional algorithm, this paper proposes the adaptive genetic algorithm [17], in which probabilities of crossover and mutation can change adaptively according to individual fitness. The adaptive change will maintain the diversity of population, improve the capability of global search, and avoid individual being mature earlier,

$$
\begin{aligned}
& P_{c}= \begin{cases}P_{c 1}-\frac{\left(P_{c 1}-P_{c 2}\right)\left(f^{\prime}-f_{\mathrm{avg}}\right)}{f_{\mathrm{max}}-f_{\mathrm{avg}}}, & f^{\prime} \geq f_{\mathrm{avg}}, \\
P_{c 1}, & f^{\prime}<f_{\mathrm{avg}},\end{cases} \\
& P_{m}= \begin{cases}P_{m 1}-\frac{\left(P_{m 1}-P_{m 2}\right)\left(f-f_{\mathrm{avg}}\right)}{f_{\max }-f_{\mathrm{avg}}}, & f \geq f_{\mathrm{avg}}, \\
P_{m 1}, & f<f_{\mathrm{avg}},\end{cases}
\end{aligned}
$$

where $P_{c}$ is the crossover probability, $P_{m}$ is the mutation probability, $f_{\max }$ is the maximum fitness of population, $f_{\mathrm{avg}}$ is the average fitness, $f^{\prime}$ is the larger fitness of two individuals in crossover, and $f$ is the fitness of individual in mutation. Based on repeated experiments and former experience, the paper chooses $P_{c 1}=0.9, P_{c 2}=0.7, P_{m 1}=0.1$, and $P_{m 2}=0.002$.

3.2. LSSVM Algorithm. LSSVM was proposed by Suykens et al. [18] to train an SVM by solving a set of linear equations. The primary differences between LSSVM and SVM are that LSSVM transforms the inequality constraints into equality constraints and employs a square instead of the empirical risk quadratic. LSSVM can be written as follows [19]:

$$
\begin{aligned}
& \min _{w, b, e} J(w, e)=\frac{1}{2}\left(w^{T} w\right)+\frac{1}{2} C\left(\sum_{i=1}^{n} e_{i}^{2}\right) \\
& \text { S.T. } y_{i}=w^{T} \phi\left(x_{i}\right)+b+e_{i} .
\end{aligned}
$$

Here, $w^{T} \phi\left(x_{i}\right)$ is the linear classifier in the feature space, $b$ is the bias parameter, $e_{i}$ is the error of the $i$ th training example, such that $\sum_{i=1}^{n} e_{i}^{2}$ is the empirical risk, and $\sum_{i=1}^{n} e_{i}^{2}$ represents the penalty factor. We can then acquire the Lagrange function

$$
\begin{aligned}
L(w, b, e, a)= & J(w, e) \\
& -\sum_{i=1}^{n} a_{i}\left\{w^{T} \phi\left(x_{i}\right)+b+e_{i}-y_{i}\right\},
\end{aligned}
$$

where $a_{i}$ is the Lagrange multipliers.
The following are established according to the KarushKuhn-Tucker (KKT) condition:

$$
\begin{aligned}
& \frac{\partial L}{\partial w}=0 \longrightarrow w=\sum_{i=1}^{n} \alpha_{i} \phi\left(x_{i}\right), \\
& \frac{\partial L}{\partial b}=0 \longrightarrow \sum_{i=1}^{n} \alpha_{i}=0, \\
& \frac{\partial L}{\partial e_{i}}=0 \longrightarrow \alpha_{i}=C e_{i}, \\
& \frac{\partial L}{\partial a_{i}}=0 \longrightarrow y_{i}=w^{T} \phi\left(x_{i}\right)+b+e_{i} .
\end{aligned}
$$

By eliminating the parameters $e$ and $w$ in (10), the equation can be rewritten as

$$
g(x)=\sum_{i=1}^{n} \alpha_{i} y_{i} d\left(x_{i}, x\right)+b .
$$

The kernel function in this paper adopts the radial basis function

$$
d\left(x_{i}, x\right)=\exp \left(-\frac{\left\|X-X_{K}\right\|^{2}}{2 \sigma^{2}}\right),
$$

where $\sigma$ is the kernel width.

3.3. Selection of LSSVM Parameters by GA. After building the LSSVM model, GA is carefully designed to optimize the penalty factor and kernel parameters of LSSVM, avoiding premature convergence and permutation problems. The GALSSVM involves several steps as follows.

Step 1 (encoding and initialization). Free parameters $c$ and $\sigma$ are represented by a chromosome comprised of two genes.

Step 2 (calculating fitness function). A fitness function is used to assess the quality of a solution.

Step 3 (parent selection). Two chromosomes with higher fitness values are selected from the parent population.

Step 4 (crossover and mutation). Crossover randomly exchanges genes between two chromosomes, and the mutation operator occasionally converts a " 1 " bit into a " 0 " bit or vice versa within a candidate solution's genes.

Based on the algorithm elements described above, a flowchart of the proposed method for railway rolling bearing fault diagnosis using STWD-EMD-GA-LSSVM is presented in Figure 1. As shown in the flowchart, the raw vibration signal is denoised by STWD, EMD is used to decompose the denoised signal into a number of IMFs, and the IMF energytorques are calculated. The GA is then used to optimize the LSSVM, and, finally, the GA-LSSVM is used for classification of the feature parameters. 


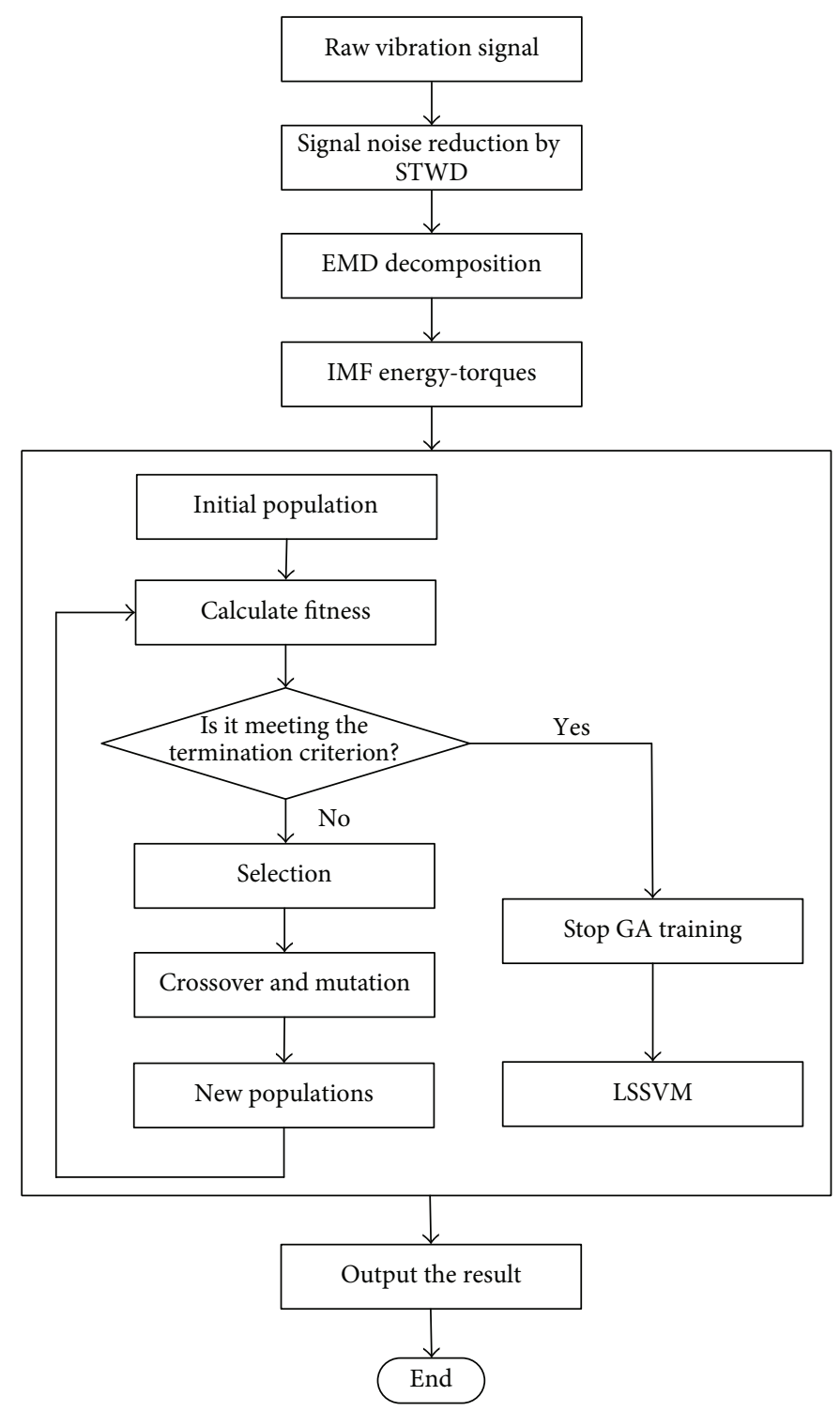

FIGURE 1: Flowchart of the novel intelligent fault diagnosis model.

\section{Experimental Results}

4.1. Experimental Setup. The experiments were performed on a SpectraQuest, Inc., machinery fault simulator capable of simulating a range of machine faults such as gearbox, shaft misalignment, rolling element bearing, and resonance and reciprocating mechanism effects and the experimental setup is shown in Figure 2. It consists of a variable speed drive, a parallel shaft gearbox with rolling bearings, and a programmable magnetic brake (for gearbox loading). Vibration signals were collected with $12 \mathrm{kHz}$ sampling rate for three different conditions under a given motor loading: (1) normal; (2) outer race fault (ORF); and (3) inner race fault (IRF).

4.2. Application. The time domain signals of the ORF are presented in Figure 3, whereas Figure 4 displays the denoised signals. Equidistant impulses are clearly observable in Figure 4,

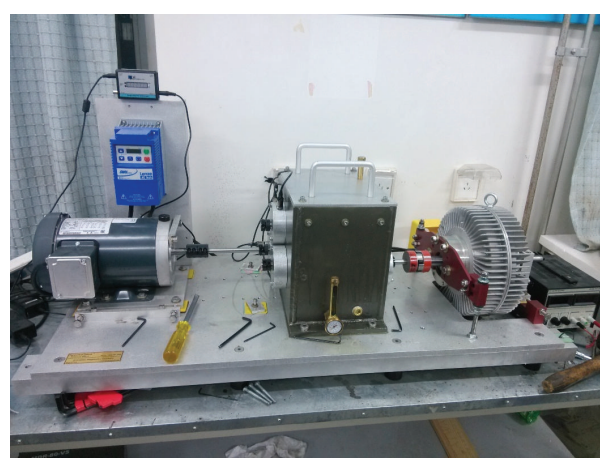

FIGURE 2: Experimental setup for bearing fault diagnosis.

in contrast to the signal given in Figure 3. Figure 5 presents 5 IMFs derived by EMD from the railway rolling bearing 


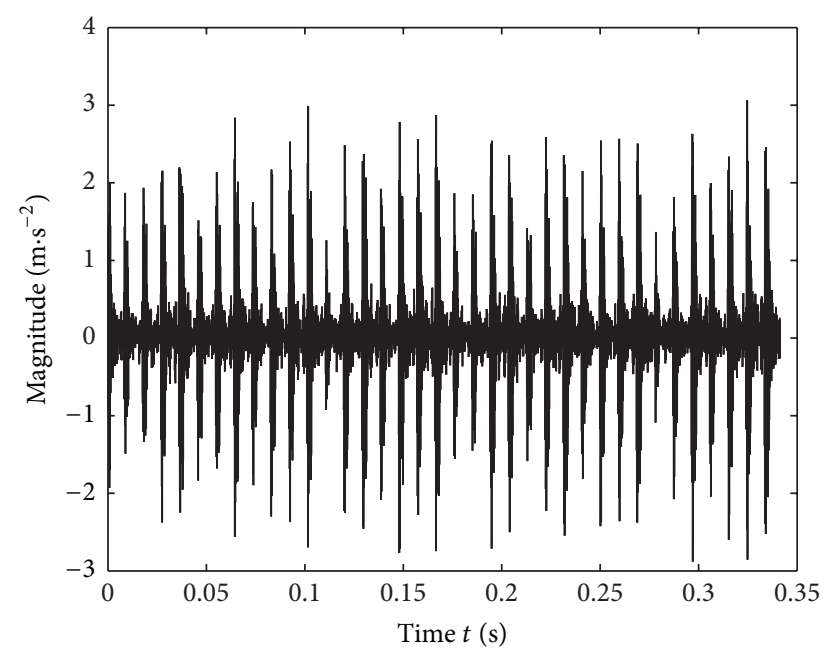

Figure 3: The time domain of the outer ring fault signal.

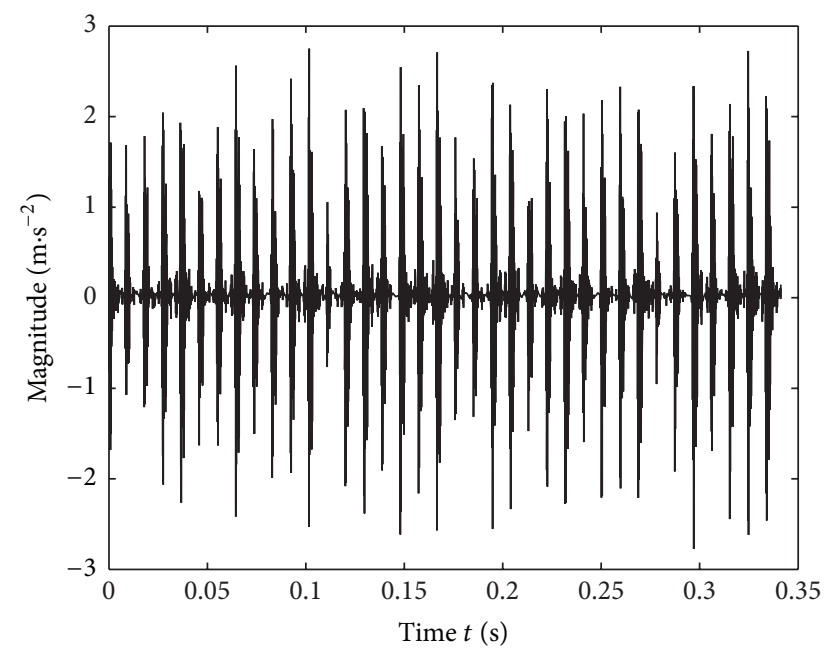

FIGURE 4: The denoised outer ring fault signal.

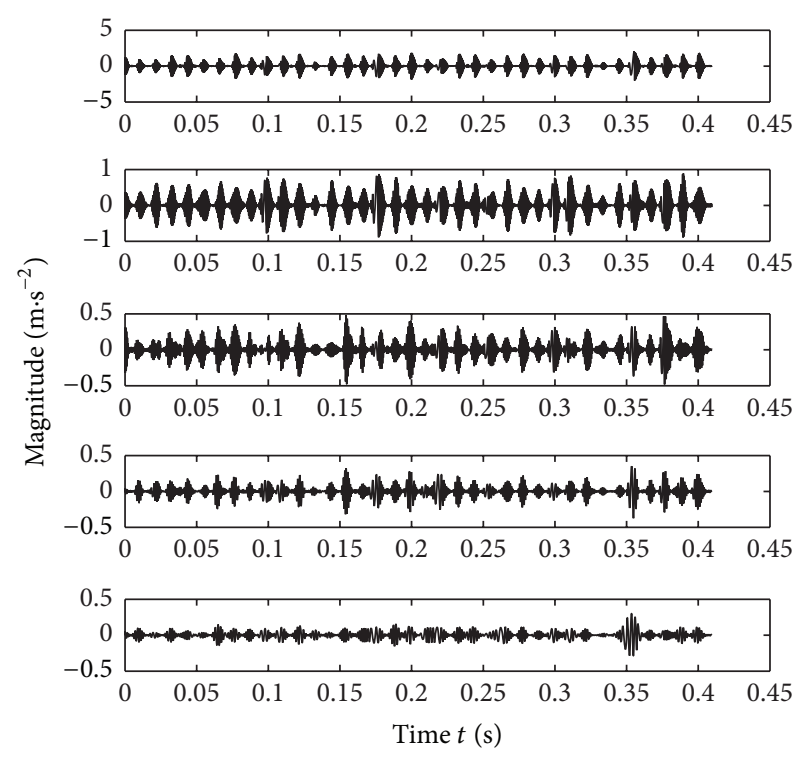

FIGURE 5: The outer ring fault signal decomposed into 5 IMFs by EMD.

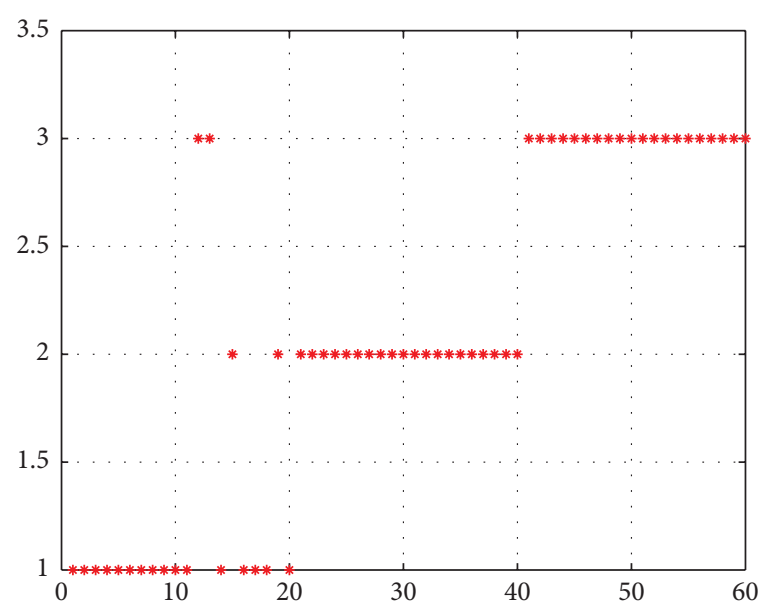

Figure 6: Testing results of the LSSVM without GA.

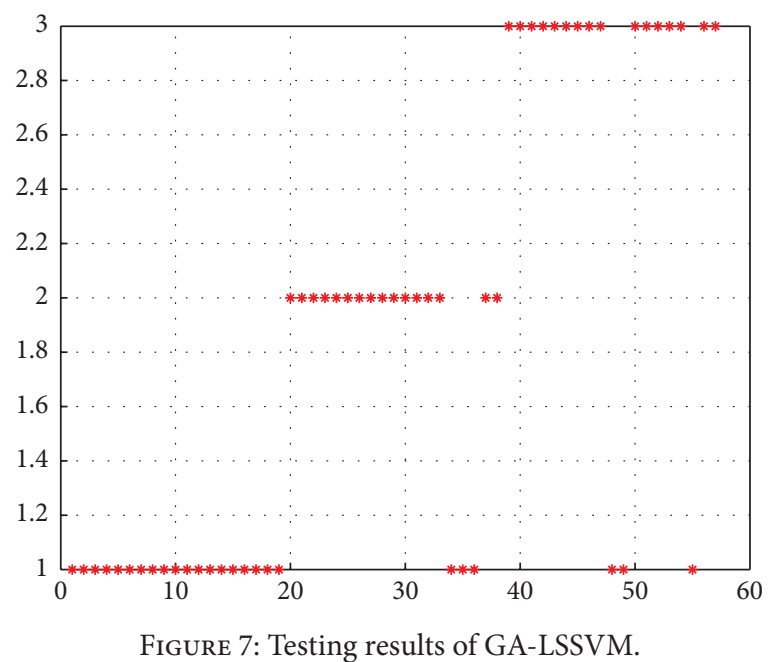

Figure 7: Testing results of GA-LSSVM. vibration signals. The energy-torque feature parameters were divided into a training group and a testing group. A total of 20 experimental datasets were obtained for each operational condition, and 15 datasets were employed for training and the remaining 5 datasets were used to test the recognition rate of the proposed method. A portion of the operational data employed for training is presented in Table 1, and a portion of the operational data used for testing is presented in Table 2. Finally, the energy-torque feature parameters are applied as input vectors of GA-LSSVM for classification, and the results are shown in Figure 7.

From Figures 6 and 7, the hybrid GA-LSSVM model obtains a higher detection rate than LSSVM for fault recognition of railway rolling bearings. The experimental results verify that the proposed STWD-EMD-GA-LSSVM method is useful for classifying the railway rolling bearings faults considered. 
TABLE 1: A portion of the bearing operational data employed for training.

\begin{tabular}{ccccccc}
\hline & $E_{1}$ & $E_{2}$ & $E_{3}$ & $E_{4}$ & $E_{5}$ & Fault status \\
\hline 1 & 0.9766 & 0.1272 & 0.0991 & 0.122 & 0.0737 & Normal signal \\
2 & 0.9596 & 0.148 & 0.077 & 0.209 & 0.0873 & Normal signal \\
3 & 0.9562 & 0.1429 & 0.0679 & 0.2365 & 0.0678 & Normal signal \\
4 & 0.8743 & 0.4503 & 0.113 & 0.0959 & 0.1043 & Inner ring fault signal \\
5 & 0.8929 & 0.4201 & 0.0986 & 0.1107 & 0.0649 & Inner ring fault signal \\
6 & 0.8792 & 0.4137 & 0.188 & 0.1122 & 0.0892 & Inner ring fault signal \\
7 & 0.9711 & 0.1659 & 0.0222 & 0.0346 & 0.1667 & Outer ring fault signal \\
8 & 0.9631 & 0.1525 & 0.1587 & 0.0216 & 0.1532 & Outer ring fault signal \\
9 & 0.951 & 0.2013 & 0.2162 & 0.0186 & 0.0895 & Outer ring fault signal \\
\hline
\end{tabular}

TABLE 2: A portion of the bearing operational data used for testing.

\begin{tabular}{ccccccc}
\hline & $E_{1}$ & $E_{2}$ & $E_{3}$ & $E_{4}$ & $E_{5}$ & Fault status \\
\hline 1 & 0.9772 & 0.1131 & 0.1109 & 0.1171 & 0.079 & Normal signal \\
2 & 0.9745 & 0.1355 & 0.0859 & 0.1415 & 0.067 & Normal signal \\
3 & 0.9682 & 0.1313 & 0.1311 & 0.1566 & 0.0605 & Normal signal \\
4 & 0.8771 & 0.421 & 0.1522 & 0.1472 & 0.0926 & Inner ring fault signal \\
5 & 0.9034 & 0.3704 & 0.1352 & 0.1479 & 0.0806 & Inner ring fault signal \\
6 & 0.7942 & 0.5615 & 0.1654 & 0.099 & 0.1296 & Inner ring fault signal \\
7 & 0.9446 & 0.2831 & 0.0192 & 0.0163 & 0.1642 & Outer ring fault signal \\
8 & 0.9476 & 0.1171 & 0.272 & 0.0159 & 0.119 & Outer ring fault signal \\
9 & 0.9443 & 0.1283 & 0.2912 & 0.0493 & 0.068 & Outer ring fault signal \\
\hline
\end{tabular}

\section{Conclusions}

To improve the signal-to-noise ratio, a novel method for the fault diagnosis of railway rolling bearings using STWDEMD-GA-LSSVM was presented. Firstly, the raw vibration signal was denoised using STWD. Then, EMD was used to decompose the signal, and the IMF energy-torques were extracted as feature parameters. A GA was carefully designed to optimize the LSSVM, avoiding premature convergence and permutation problems. Finally, fault samples of IMF energytorques were used as LSSVM input parameters to realize intelligent fault diagnosis. The testing results show that the proposed approach can effectively and accurately diagnose railway rolling bearing faults.

\section{Competing Interests}

The authors declare that they have no competing interests.

\section{Acknowledgments}

This paper was supported by the National Natural Science Fund Project (51175028), the Research of Bearing Fault Mechanism and Identification Algorithm for Urban Rail Train Running Gear (00331615015), and the International Science \& Technology Cooperation Program of China (2014DFR70280).

\section{References}

[1] D. Yao, L. Jia, Y. Qin, and J. Yang, "Faults diagnosis of railway rolling bearing by using time-frequency feature parameters and genetic algorithm neural network," Computer Modelling and New Technologies, vol. 18, no. 10, pp. 441-445, 2014.

[2] M. N. Khajavi and M. N. Keshtan, "Intelligent fault classification of rolling bearings using neural network and discrete wavelet transform," Journal of Vibroengineering, vol. 16, no. 2, pp. 761769, 2014.

[3] B. Wang, S. L. Liu, H. L. Zhang, and C. Jiang, "Fault diagnosis of rolling bearing based on relevance vector machine and kernel principal component analysis," Journal of Vibroengineering, vol. 16, no. 1, pp. 57-69, 2014.

[4] L. Auslander, "Comparison of the Gabor and short-time Fourier transforms for signal detection and feature extraction in noisy environments," The International Society for Optical Engineering, vol. 13, no. 48, pp. 230-247, 1990.

[5] K. Feng, Z. Jiang, W. He, and B. Ma, "A recognition and novelty detection approach based on Curvelet transform, nonlinear PCA and SVM with application to indicator diagram diagnosis," Expert Systems with Applications, vol. 38, no. 10, pp. 12721-12729, 2011.

[6] J.-D. Wu and C.-H. Liu, "An expert system for fault diagnosis in internal combustion engines using wavelet packet transform and neural network," Expert Systems with Applications, vol. 36, no. 3, pp. 4278-4286, 2009.

[7] B. Samanta, "Artificial neural networks and genetic algorithms for gear fault detection," Mechanical Systems and Signal Processing, vol. 18, no. 5, pp. 1273-1282, 2004. 
[8] J. Dybała and R. Zimroz, "Rolling bearing diagnosing method based on empirical mode decomposition of machine vibration signal," Applied Acoustics, vol. 77, pp. 195-203, 2014.

[9] N. E. Huang, Z. Shen, S. R. Long et al., "The empirical mode decomposition and the Hilbert spectrum for nonlinear and non-stationary time series analysis," Proceedings of the Royal Society of London Series A: Mathematical and Physical Sciences, vol. 454, no. 1971, pp. 903-995, 1998.

[10] I. Obuchowski, A. Wyłomańska, and R. Zimroz, “The local maxima method for enhancement of time-frequency map and its application to local damage detection in rotating machines," Mechanical Systems and Signal Processing, vol. 46, no. 2, pp. 389405, 2014.

[11] G. F. Bin, J. J. Gao, X. J. Li, and B. S. Dhillon, "Early fault diagnosis of rotating machinery based on wavelet packetsempirical mode decomposition feature extraction and neural network," Mechanical Systems and Signal Processing, vol. 27, no. 1, pp. 696-711, 2012.

[12] J. H. Holland, Adaptation in Natural and Artificial Systems, University of Michigan Press, Ann Arbor, Mich, USA, 1975.

[13] C.-L. Huang and C.-J. Wang, "A GA-based feature selection and parameters optimizationfor support vector machines," Expert Systems with Applications, vol. 31, no. 2, pp. 231-240, 2006.

[14] J. Mendes, R. Araújo, T. Matias, R. Seco, and C. Belchior, "Automatic extraction of the fuzzy control system by a hierarchical genetic algorithm," Engineering Applications of Artificial Intelligence, vol. 29, pp. 70-78, 2014.

[15] E. Kahya, "A new unidimensional search method for optimization: linear interpolation method," Applied Mathematics and Computation, vol. 171, no. 2, pp. 912-926, 2005.

[16] Y.-P. Zou and H.-L. Liu, "New dynamic load balancing method based on roulette wheel selection and its implementation," Journal on Communications, vol. 29, no. 9, pp. 18-23, 2008.

[17] X. L. Ren, X. F. Chen, and J. Ma, "Genetic algorithms and BP neural networks used in traffic flow forecasting," Technology and Economy in Areas of Communications, vol. 54, no. 4, pp. 10-12, 2009.

[18] Y. Wang and H. Fu, "Parameters selection of LSSVM based on adaptive genetic algorithm for ship rolling prediction," in Proceedings of the 33rd Chinese Control Conference (CCC '14), pp. 6632-6636, IEEE, Nanjing, China, July 2014.

[19] J. A. K. Suykens, J. Vandewalle, and B. De Moor, "Optimal control by least squares support vector machines," Neural Networks, vol. 14, no. 1, pp. 23-35, 2001. 


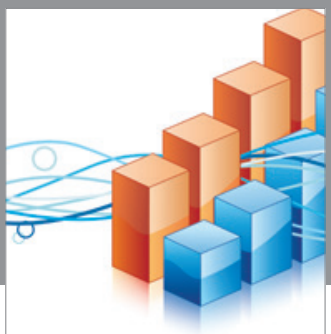

Advances in

Operations Research

vatem alat4

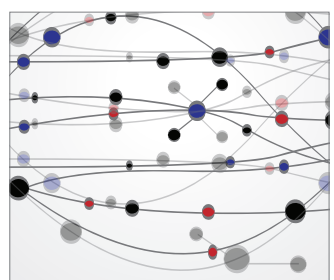

\section{The Scientific} World Journal
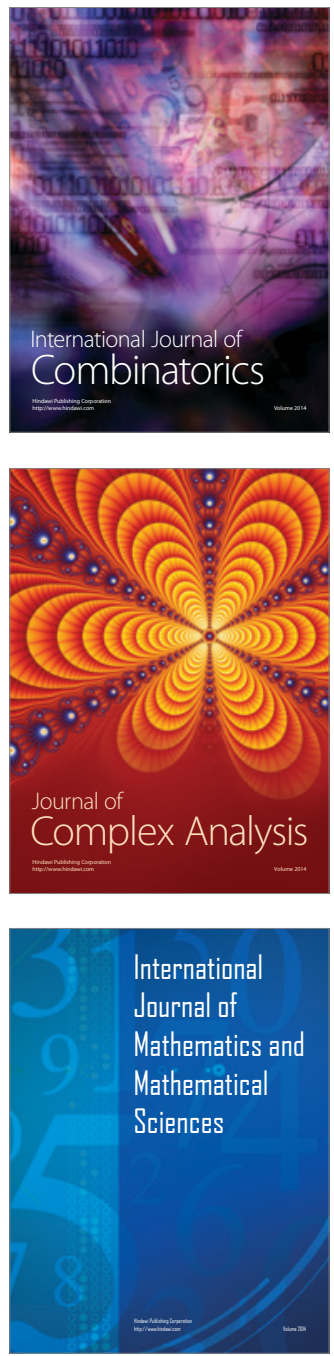
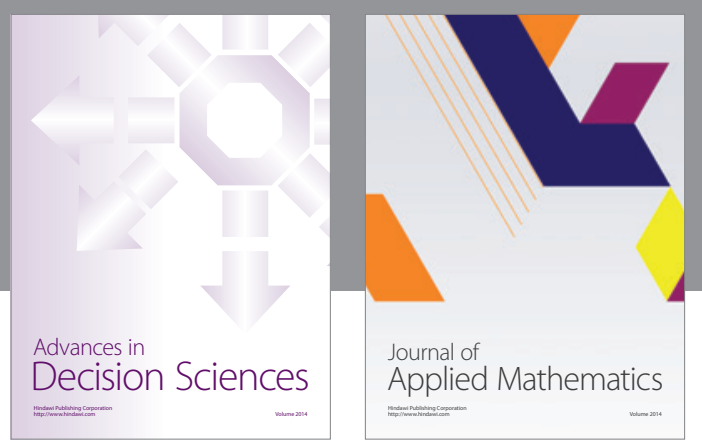

Algebra

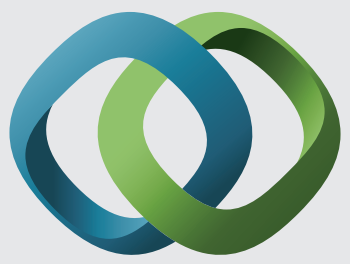

\section{Hindawi}

Submit your manuscripts at

http://www.hindawi.com
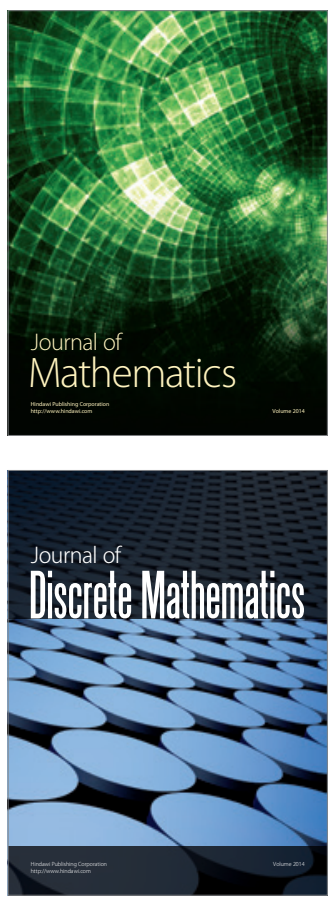

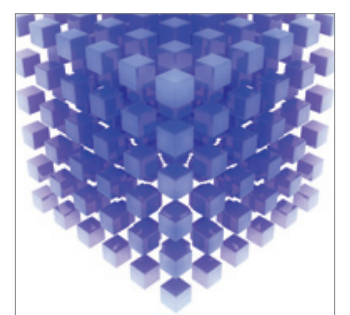

Mathematical Problems in Engineering
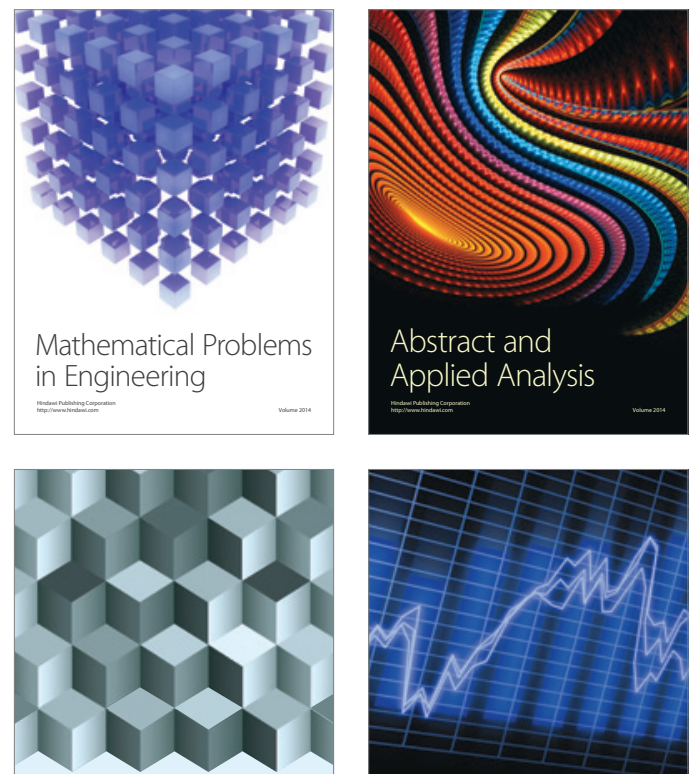

Journal of

Function Spaces

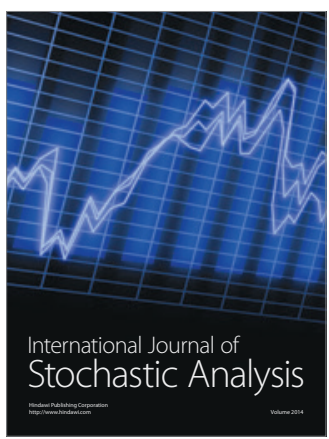

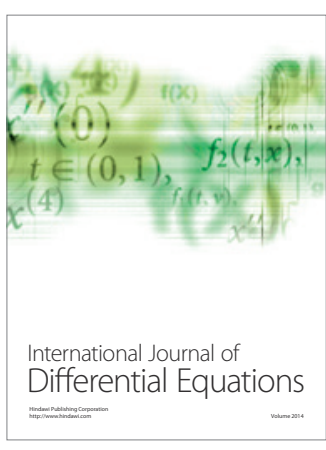
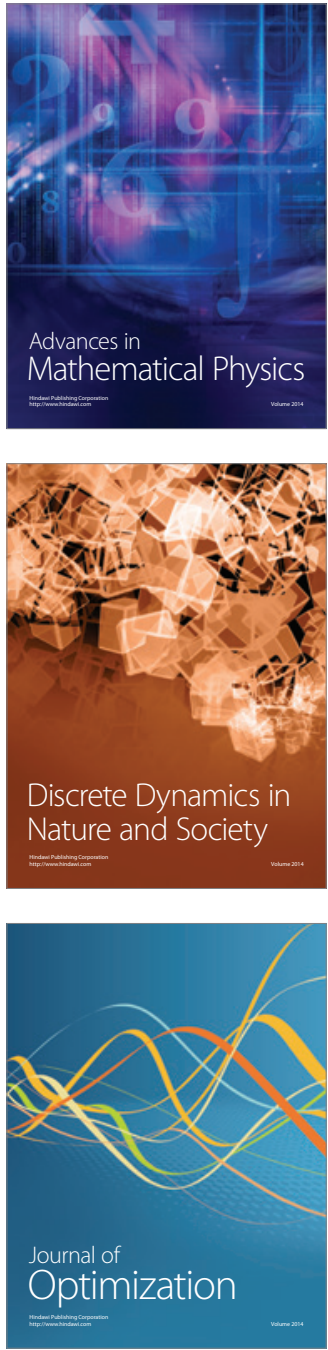